\title{
XIII. Geometrical interpretation of log Uq
}

\section{Alexander Macfarlane}

To cite this article: Alexander Macfarlane (1894) XIII. Geometrical interpretation of log Uq , Philosophical Magazine Series 5, 38:230, 143-144, DOI: 10.1080/14786449408620610

To link to this article: http://dx.doi.org/10.1080/14786449408620610

$$
\text { 曲 Published online: } 08 \text { May } 2009 .
$$

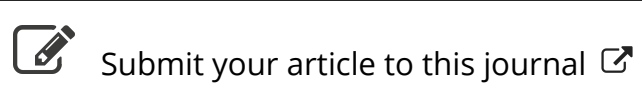

\footnotetext{
Џll Article views: 2
}

Q View related articles ¿ 


\section{[ 143$]$ \\ XIII. Geometrical Interpretation of $\log \mathrm{U} q$. By Alexander Macfartane.}

To the Editors of the Philosophieal Magazine.

\section{GeNTLEMEN,}

$\mathrm{T}^{\mathrm{N}}$ the notice of Dr. Molenbroek's Anwendung der Qua1 ternionen auf der Geometrie, your reviewer says, "It would probably baffle even a Hamilton to give a geometrical interpretation of $\log \mathrm{U} q "$ (Phil. Mag. vol. xxxvii. p. 333). As this matter has been treated of in several of my papers, $\mathbf{I}$ send you the interpretation required.

The general quaternion $q$ may be analysed into the product of a ratio and a versor; by $\mathrm{U} q$ is meant the versor. Let a denote the axis of the versor and $\mathrm{A}$ its amount in radians, then $\mathrm{U}_{q}=\alpha^{\mathrm{A}}$ and $\log \mathrm{U}_{q}=\mathrm{A} \alpha^{\prime}$; hut $\log \alpha^{\prime}=\alpha^{\frac{\pi}{2}}$, therefore $\log \mathrm{U}_{q}=\mathrm{A} \alpha^{\frac{\pi}{2}}$. A more correct definition of $\mathrm{A}$ is the ratio of twice the area of the sector to the square of the initial radius; for that definition applies also to a hyperbolic versor.

The geometrical meaning of the above expression will become evident on considering the more general versor given by an equiangular spiral. Let a quinion be denoted by $q^{\prime}$, and let it be defined to be such that

$$
\log \mathrm{U} q^{\prime}=\mathrm{A} \alpha^{w}=\mathrm{A} \cos w+\mathrm{A} \sin w \cdot \alpha^{\frac{\pi}{2}} ;
$$

we then find that $A \sin w \cdot \alpha^{\frac{\pi}{2}}$ is the logarithm of the angle and $\mathrm{A} \cos w$ the logarithm of the radius of an equiangular spiral of axis $\alpha$ and constant angle $w$, the initial radius being unity. Thus $w$ is the constant angle between the radiusvector and the tangent, or rather the difference of the angle from the initial radius to the tangent and that from the initial radius to the radius-vector. In the case of the circle this difference angle is a quadrant: this is the explanation of the quadrantal versor in $\log \mathrm{U} q$. In the spiral the quantity $\mathrm{A}$ is the magnitude of the complex logarithm, and $\alpha^{w}$ gives the components of the logarithm. The expansion depends on the scalar component of the logarithm, while the rotation depends on the vector component. In the case of the circle, that is of $\mathrm{U} q$, the scalar logarithm vanishes.

For further elucidation of this matter consider a hyperbolic quaternion. Let $p$ denote such a quaternion; when the multiplier is removed we have $\mathrm{U}_{p}=\alpha^{i \mathrm{~A}}$ and therefore $\log \mathrm{U}_{p}=i \mathrm{~A} \alpha^{\frac{\pi}{2}}$. 
Here $A$ is the ratio of twice the area of the hyperbolic sector to the square of the initial radius, and $a^{\frac{\pi}{2}}$ is the constant sum of the angle from the initial radius to the tangent and that from the initial. radius to the radius-vector. This leads us to consider the hyperbolic analogue of the equiangular spiral. Let $p^{\prime}$ denote the corresponding hyperbolic quinion; then

$$
\log \mathrm{U} p^{\prime}=i \mathrm{~A} \cdot \alpha^{w}=i \mathrm{~A}\left(\cos w+\sin w \cdot \alpha^{\frac{\pi}{2}}\right) .
$$

Here $w$ denotes the constant sum of the angle between the initial vector and the radius-vector and the angle between the initial vector and the tangent. The scalar term $i \mathrm{~A} \cos w$ is the logarithm of the radius-vector, while the vector term $i \mathrm{~A} \sin w \cdot \alpha^{\frac{\pi}{2}}$ is the logarithm of the hyperbolic angle. Here $i$ is the scalar $\sqrt{-1}$; in the papers on "The Principles of Elliptic and Hyperbolic Analysis," and on "The Definitions of the Trigonometric Functions," I have shown that a quantity which is the sum of a scalar independent of $i$ and another scalar dependent on $i$ is represented along one straight line.

\author{
University of Texas, \\ Austin, Texas, U.S.A., \\ May 10, 1894 .
}

Alexander Mactarlane.

XIV. On a Fundamental Question in Electro-Optics.

To the Editors of the Philosophical Magazine.

\title{
Gentilemen,
}

WILL you kindly afford me space for a few remarks in connexion with Professor Quincke's letter, which appeared in the May number of this Magazine. The object of that letter was to draw attention to the fact that, in my paper on Electro-Optics which appeared in the April number, I made no mention of a paper of Professor Quincke's, published eleven years ago, which gives an account of experiments by him on the same subject and by similar methods. I think I should preface what I have to say on this matter with an expression of regret for my forgetfulness.

I was well aware of the existence of that paper. I received a copy from the author, and perused it I think immediately on receipt. With regard to the electro-optic effects there described, as given by an interference-refractor, it was evident to me then, as it is now, that they were in their nature and immediate origin essentially different from those pure double refractions that are given regularly by the common polari- 